\title{
A New Simplified PDF Method for Calculating Major Species Concentrations and Burning in Turbulent Fires
}

\author{
M. A. DELICHATSIOS and M. K. MATHEWS \\ Factory Mutual Research Corporation \\ Norwood, Massachusetts 02062, USA
}

ABSTRACT

A simplified method is presented for calculating mean major species concentrations $\left(\mathrm{O}_{2}, \mathrm{CO}_{2}, \mathrm{CO}\right)$ in turbulent fires for any fuel given the species distribution of the same fuel in a laminar diffusion flame. This method, verified by extensive measurements, uses a probability distribution function ( $P$ df) for the conserved scalar in turbulent fires together with a uniform mixedness parameter throughout the flames, first proposed

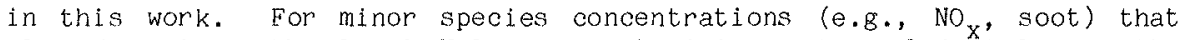
also depend on the local Kolmogorov straining rates of turbulence, the present method might be extended by including the distribution of turbulent straining rates in a fashion strongly suggested by recent evidence of soot-flame radiation and $\mathrm{NO}_{x}$ yield in turbulent diffusion flames. Finally, based on the present method, an integral model is outlined for turbulent buoyant jet flames.

\section{CHEMICAL SPECIES IN TURBULENT FIRES}

It is surprising that data obtained by $L$. Orloff $(1)$ on mean major species concentrations in turbulent pool fires can be correlated with the mean corresponding mixture fraction independently of 1) location inside the fire plume, 2) the total heat release rate, or 3) the diameter of the fire. Figure 1( $a, b, c)$ illustrates this result notwithstanding the scatter close to the maximum concentration values. Figure 1 also includes the corresponding data for the opposing laminar flame of Tsuji and Yamaoka (2). Plotting the experimental data in terms of the coordinates of Figure 1 implies 1) equal diffusivities and 2) chemical reactions unaffected by the straining action of the flowfield. The average elemental fraction or mixture fraction used as abscissa in Figure 1 is a conserved quantity in the fire and represents, for example, the ratio of carbon atom mass at a given place relative to the carbon atom mass at the source ${ }^{1)}$.

If the previous assumptions are accepted, the results of L. Orlof ( 1 ) as illustrated in Eigure 1 suggest the possibility of predicting the major species concentrations in turbulent fires by using a simple mixing and combustion model.

Eirst, we observe that turbulent buoyant flames generate large eddies that move rather lazily inside the fire plume. The visible Kolmogorov miorot scale is about $1-2 \mathrm{~cm}$. The diffusivity is of order of $2 \mathrm{~cm}^{2} / \mathrm{sec}$ so that the Kolmogorov time scale $\tau_{K} \sim \frac{\ell^{2}}{D}$ is of the order of a second. This time 
scale is quite insensitive to the heat release rate or the flame siz For common fuels, the reaction times are much smaller than the Kolmogor time scales characteristio for turbulent buoyant fires. It follows th the reaction zones are also much smaller than the Kolmogorov microsca and thus, the instantaneous diffusion flame structure is unaffected by $t$ transient nature of the turbulent field.* In addition, experiments ha established that, away from extinction conditions, the chemical structu within laminar diffusion flames is quite insensitive to their loc straining rates or flow times, so that the concentration of major stab species is a unique function of the mixture fraction, $\xi: Y_{S}=Y_{S}(\xi)$ (cf with Figure 1). Therefore, we may conclude that in turbulent, fir the instantaneous concentration of a stable species should be equal to 1 value corresponding to laminar diffusion flame conditions.

Such a simple relationship suggests that one can predict the mean speci concentrations in turbulent fires if the probability distribution, $P(\xi$ of the random varying value of the mixture fraction, $\xi$, is known, i.e.

$\bar{Y}_{S}(\bar{\xi})=\int_{0}^{1} Y_{S, L}(\xi) P\left(\xi, \bar{\xi}, \overline{\xi^{\prime 2}}\right) d \xi$

where we assume that $P(\xi)$ is functionally dependent on the average val of $\bar{\xi}$ and its mean square fluctuations $\overline{\bar{\xi}^{\prime 2}}$.

It is difficult to predict or measure the probability distribution fun tion of the conserved scalar, $\xi$, notwithstanding the recent advances this area(4). (Two areas not yet satisfactorily modeled are: a) $t$ dissipation and mixing of a conserved scalar, and b) the velocity-co centration correlations $\left.\overline{u^{\prime} \xi^{\prime}}\right)$. Instead, we used a pdf for the conserv scalar having parameters that match measured (or predicted) values of $t$ mean mixture fraction $\bar{\xi}$ and its fluctuations $\xi^{\prime 2}=(\xi-\bar{\xi})^{2}$. In genera the pdf of a conserved scalar has the following form:

$P(\xi)=\alpha \delta(\xi)+\beta P_{t}(\xi)+\gamma \delta(1-\xi)$ where $0 \leqq \xi \leqq 1$

Here $\alpha$ and $\gamma$ represent fractions of the yet unmixed constituents $(\xi=$ for air; $\xi=1$ for fuel); $B$ is the fraction of the gas mixed turbulence; finally $P_{t}(\xi)$ is the Pdf of the turbulence mixed part:

$\int P_{t}(\xi) d \xi=1 .(0 \leqq \xi \leqq 1)$. (Note that $\left.\alpha+\beta+\gamma=1.\right)$ The shape the turbulent part $\beta P_{t}\left(\xi_{0}\right)$ can be that of an arbitrary function clipped $\xi=0$ and $\xi=1$. Figure 2 il.lustrates the general form of the probabili distribution function. For a beta-pdf the spikes at $\xi=0$ and at $\xi=1$ a included in the form of the beta-function:

$\beta(x)=\frac{\Gamma(\lambda)}{\Gamma(n)} \overrightarrow{\Gamma(m)} \xi^{n-1}(1-\xi)^{m-1}$

where $\Gamma()$ is the classical $\Gamma$-function and the parameters $\lambda, n, m a$ related to the average value of $\bar{\xi}$ and its fluctuations by:

* If the reaction time and hence, the reaction zone are greater than corresponding Kolmogorov scales, the structure of the instantaneous $c$ fusion flame will be affected by the turbulent flow. It is difficult describe the combustion in such flows. Fuel eddies with a life $t$ (which is reciprocal to their characteristic strain rate) larger than reaction time will sustain regular diffusion flames, while fuel edc with a life time much less than the reaction-time will extinguish and directly with the air without sustaining combustion. 
$n=\lambda \bar{\xi}$

$m=\lambda(1+\bar{\xi})$

$\lambda=\frac{\bar{\xi}(1-\bar{\xi})}{\bar{\xi}^{1^{2}}}-1$

We used different forms of the "turbulent" Pdf, $P_{t}(\xi)$, (e.g. Beta, Gaussian) to predict the mean species concentrations based on eq. $1^{*}$. To complete the calculations, one has to select a value for the level of fluctuations $\overline{\xi^{\prime 2}}$ (all "turbulent" pdf's used here are two parameter functions). The appropriate parameter is the relative mixedness ratio:

$$
\varepsilon=\frac{\overline{\xi^{12}}}{\bar{\xi}(1-\bar{\xi})} \leqq 1
$$

which is always less than one. Note that the denominator of eq. 4 represents the (maximum) level of fluctuations in the absence of molecular mixing. In general, the mixedness ratio (eq. 3 ) is a function of $\bar{\xi}$; however, a fixed value throughout the flames proved to adequately describe the data. In fact, the mixedness parameter $\varepsilon$ for a fuel jet would decrease along the fire plume from a value one near the nozzle to the asymptotic value of zero far from the source after combustion is completed (note that for the farmield plume $\frac{\frac{\xi^{1^{2}}}{\bar{\xi}^{2}}}{\text { constant). }}$.

The results of the present analysis are compared in Figures $3(a, b, c)$ and $4(a, b, c)$ with the experimental data, The laminar data of the opposing diffusion flame of Tsuji and Yamaoka $(2)$, as shown in Figure $1(a, b, c)$, were used as state functions $Y_{s, L}(\xi)$ in eq. (1) to caloulate the species concentrations in turbulent fires. Note that the experimental data of Tsuji and Yamaoka do not include effects of radiation cooling which is minimal in an opposing flow but reaches high values in turbulent buoyant flames (about 30\% for propane). It is apparent that the shape of the "turbulent" part of the Pdf does not affect the results significantly: a beta pdf was used for the predictions shown in Figure 3 and a clipped Gaussian in Figure 4. The best fit for all species (shown in Figures 3

and 4) was obtained for a value of the mixedness ratio

$$
\varepsilon=\frac{\overline{\xi^{\prime 2}}}{\bar{\xi}(1=\bar{\xi})}=
$$

.11 , which represents a rather large degree of molecular mixing (note that $\varepsilon=1$ in the absence of molecular mixing while $\varepsilon=0$ for complete molecular mixing). There are no direct measurements of the mixedness parameter or the fluctuations of the mixture fraction in axisymmetric turbulent buoyant, jets. Caloulations based on $k^{-}-\varepsilon^{-g}$ models inside the flame envelope (10) provide values consistent with the "best" estimated value here.

One may reach the following conclusions by inspection of Figures 3 and 4: 1) For fuel lean $(\bar{\xi}<.2)$ or stoichiometric conditions, the present combustion and mixing model predicts well the mean species concentration in turbulent fires based on $e q .(1)$, and a mixedness ratio $\varepsilon=.11$; 2) For fuel rich conditions, the present model underpredicts the experimental values. In fact, the experimental data for turbulent fires are higher

* The present analysis is based on the assumption that two parameters, i.e. the mean $\bar{\xi}$ and $i t s$ fluctuations are sufficient to determine the probability distribution of the conserved scalar. 
than the laminar data (of Figure 3 and 1). It is easy to show that no $p$ model (including intermittency effects) based on eq. (1) can provide me values larger than the laminar values. There is no reason to doubt $t$ accuracy of the experimental data which are consistent with similar da presented in the literature. (5) To resolve the discrepancy shown Figure 3 and 4 for $\xi>.2$, one needs to reevaluate a) the assumption equal diffusivities, b) the possibility of local extinctions due radiation cooling which may result in partially premixed conditions clo to the fire source (large $\xi$ values), and $c$ ) the use of state functio $Y_{S, L}(\xi)$ for laminar diffusion flames wherein significant radiation loss oceur.

The present method could be extended for the predictions of minor speci concentrations (e.g. soot, $\mathrm{NO}_{\mathrm{x}}$ ) that also depend on the local Kolmogor straining rates of turbulence. For this purpose a joint pdf between $t$ mixture fraction, $\xi$, and the fluctuating Kolmogorov straining rates mu be postulated. Such an approach would be consistent with recent eviden in turbulent diffusion flames $(11,12)$ : for example, measurements of $N$ yield and soot-flame radiation have been successfully correlated with t flow parameters by assuming that both mean No $x$ and soot concentrations the flames are inversely proportional to the mean straining rate inside flamelet. This straining rate is inversely proportional to the me Kolmogorov time: $\tau_{k} \sim \checkmark \bar{v}$, where $v$ is the kinematic viscosity and $\varepsilon$ the mean turbulent dissipation rate.

AN OUTLINE OF AN INTEGRAL MODEL FOR TURBULENT JET FLAMES BASED ON A GLOB PDF APPROACH

The present analysis of a global pdf for calculating species concentr tions leads to the development of an integral model for turbulent fire An outline of this model is presented in this section. For simplicity, limit the discussion to turbulent buoyant jet fires. The integr equations include: 1) the entralnment equation, 2) the momentum equatio 3) a conserved scalar equation, 4) a beta pdf for the conserved scal with a mixedness parameter (see eq. 4) $E=.11$ as suggested by the prese analysis, 5) assumed radial profiles for mean velocity and mean conserv scalar, $\bar{\xi}$.

The entrainment equation in the combustion zone, i.e. $z<z_{f}$, is

$\psi_{t}=.018 \rho_{\infty} z^{5 / 2} \frac{\overline{\Delta H_{C}}}{\left(\frac{C}{S+1}\right) C_{p} T_{\infty}} g\left(x_{A}-x_{R}\right)+\dot{m}_{f} \quad$, for $z<z_{f}$

where $\psi_{t}$ is the total flow in the plume at height $z$ less than the fla height, $Z_{f}$, from the source which provides a fuel flow rate equal to $m_{f}$; is the mass stoichiometrio ratio (air to fuel); $C_{p}$ is the specific neat ambient temperature and $\Delta H_{C}$ is the heat of combustion per mass of fue Subscript $\infty$ refers to ambient property values. Finally, $x_{A}$ and $x_{R}$ a respectively the overall combustion efficiency and radiant fraction. E (5) has been obtained from a correlation of experimental data $(6,7)$. F pool fires $(7)$ an equation different from eq. (7) must be used. strict speaking, eq. (5) has shown to be applicable ${ }^{(7)}$ for relatively lar values of the stoichiometric ratio $S>10$, which most fuels have.

The mean momentum equation is:

$\frac{d W}{d z}=\int_{0}^{\infty} g 2 \pi\left(\rho_{\infty}-\bar{\rho}\right) r d r$ 
or by employing perfect gas laws with equal molecular weight

$\frac{d W}{d Z}=2 \pi \int_{0}^{\infty} g \frac{\left(\bar{T}-T_{\infty}\right)}{T_{\infty}} \bar{\rho} r d r$

In eq. (6) $W$ is the total mean momentum inside the plume at height $z$ from the local source and $\bar{T}$ is its local mean temperature. The temperature in eq. ( $6 \mathrm{~b}$ ) can be calculated by using a global pdf via an equation similar to eq. (1), in terms of the local mean value of the conserved scalar $\xi$ :

$\bar{T}(\bar{\xi})=\int_{0}^{1} T_{L}(\xi) P\left(\xi, \bar{\xi}, \bar{\xi}^{T / 2}\right) d \xi$

one can use, for example, a beta pdf (see eq. 3) wherein the parameter $\lambda=\frac{1}{\varepsilon}-1=8.09(\varepsilon=.11)$. In eq. $(7), \mathrm{T}_{\mathrm{L}}(\xi)$ is the temperature profile obtained from a shvab-zeldovich model of laminar diffusion flames or, more desirably, from corresponding measurements (or exact numerical simulation) of laminar flames in regions without any significant radiation (e.g. near the base of a candie flame). Radlation losses are included in an average manner by multiplying the temperature rise $\left(\Delta \bar{T}=\bar{T}-T_{\infty}\right)$ calculated from eq. (7) by $\left(x_{A}-x_{R}\right)$ where $x_{R}$ is the global radiant fraction from the turbulent buoyant jet flame and $x_{A}$ is the overall combustion efficiency.

The integral at the right hand side of eq. (6b) can be calculated if the profiles of the mean scalar $\vec{\xi}$ throughout the flames are known. To implement this task, we postulate the following profiles for the mean velocity and mean mixture fraction:

$\bar{u}=\bar{u}_{c}\left(1-\frac{\psi}{\psi_{t}}\right)$

$\vec{\xi}=\vec{\xi}_{c}\left(1-\frac{\psi}{\psi_{t}}\right)$

where $\psi$ is the stream function,

$\psi=2 \pi \int_{0}^{r} \bar{u} \bar{p} r d r$

and subscript $c$ means centerline values. For partial justification, we note that these profiles (i.e. eq. ( $8 a$ ) and ( $8 b)$ ) coincide with Gaussian radial profiles for Boussinesque-type (i.e. constant density) plumes consistent with experiments.

Conservation of the conserved scalar implies:

$2 \pi \int_{0}^{\infty} \bar{\xi} \bar{u} \bar{\rho} \mathrm{rdr}=\dot{\mathrm{m}}_{\mathrm{f}}$

where $\dot{m}_{f}$ is the fuel supply rate at the source (note that $\bar{\xi}=1$ at the source). Eq. (9) where cross-correlations have been neglected, can also be written by using eq. ( 80 ) as

$$
\int_{0}^{\psi t} \bar{\xi} d \psi=\dot{m}_{\mathrm{f}}
$$

or by using the profile (8a)

$\bar{\xi}_{c} \psi_{t} \frac{1}{2}=\dot{m}_{f}$ 
or

$\bar{\xi}_{c}=\frac{2 \dot{m}_{f}}{\psi_{t}}$

$(10 c)$

wherein $\psi_{t}$, the total flow rate, is calculated by using eq. (5). Nol that eq. (10c) agrees with experimental data $(6,7)$.

We rewrite the momentum equation (6b) in terms of the stream functis variable:

$\frac{d\left(\frac{1}{2} u_{c} \psi_{t}\right)}{d Z}=\int_{0}^{\psi_{t}} g \frac{\left(\frac{\overline{\Delta T}}{T_{\infty}}\right)}{\bar{u}} d \psi$

since $W=2 \pi \int^{\psi} \bar{\rho} \bar{u}^{2} r d r=\int^{\psi} t \vec{u} d \psi$ and eq. (8a) has been applied in $t$ LHS of the momentum equation.

We now have a complete system of equations namely: eq. (5) (entrainmel equation), eq. (11) (momentum equation), with the supporting equations: : eq. (7) which provides the temperature profile for eq. (11) in terms. the mean conserved scalar which has a profile given by eqs. ( $8 b)$ and ( 10 , and b) the profile of the velocity, see eq. ( $8 a)$. The solution of the: equations allows one to calculate the centerline velocity in the plume.

Other relevant quantities can also be calculated from the present mode for example, the fuel flow rate at height $z$ :

$$
\begin{gathered}
\dot{\mathrm{m}}_{\mathrm{Y}_{F}}=\int_{0}^{\psi} \overline{\mathrm{Y}}_{\mathrm{F}}(\bar{\xi}) \mathrm{d} \psi=\psi_{\mathrm{t}} \int_{0}^{1} \overline{\mathrm{Y}}_{\mathrm{F}}\left[\frac{2 \dot{\mathrm{m}}_{\mathrm{f}}}{\psi_{\mathrm{t}}}(1-n)\right] \mathrm{d} \eta \\
n=\frac{\psi}{\psi_{t}}
\end{gathered}
$$

wherein $\bar{Y}_{F}(\bar{\xi})$ is calculated from eq (1) and $\bar{\xi}$ is given by eqs. (8a) a $(100)$. Finally, $\dot{m}_{f}$ is the fuel flow rate and $w_{t}$ is given by eq. (5). deriving eq. (12) we have neglected cross-correlation terms.

The present integral model differs from other integral models $(8,9)$ f. turbulent jet fires primarily because it prescribes the entrainme equation directiy as a function of height (see eq. (5)) and uses a glob pdf approach to calculate combustion. It should also be emphasized th top hat profiles for the properties in the flaming region cann adequately approximate the burning in turbulent fires because of $t$ strong nonlinear dependence of temperature (and buoyancy) on mixtu fraction through eq ( 7 ). The big drawback of the present model, whi also characterizes the previous models, $(8,9)$ is the neglect of cro correlation terms, especially between conserved scalar and velocit Comparison of its prediction with experimental data will verify how or tical these terms are. The present integral model could be extended predicting turbulent pool fires by using instead of eq. (5), a modifi equation for entrainment rates (see Ref. 7 ).

\section{CONCLUSIONS}

A global probability distribution function for the mixture fraction tog ther with a uniform mixedness parameter $(\varepsilon=.11)$ have been used correlate and predict species concentration in turbulent fires. The predictions are in good agreement with experiments for lean fuel cond 
tions (mixture fraction $\xi<.2$ ) and in fair agreement for rich fuel con ditions $(\xi>2)$. Based on this development, we have proposed a new self consistent integral model for turbulent buoyant jet flames which has the following innovations: 1 ) an entrainment equation (see eq. (5)) prescribed in terms only of the height $Z$ and fuel properties based on previous experimental data, and 2) the global pdf method for species concentrations used to characterize combustion. The model predictions should be checked with experimental data to investigate to what degree neglect of crosscorrelation terms in the model affects its validity.

We caution that the calculation of species concentrations (see eq. 1 and discussion following eq. 4) relies on the assumption that radiation losses do not significantly alter the state relationships, $Y_{S}, L$, for the laminar flamelets. Such an assumptions is a good approximation for total radiant fractions, $x_{R} \leq 30 \%$, as various measurements of $C O$, and soot overall yield have shown. However, fluctuations of the radiant fraction together with its spatial variations may result in local flame extinctions (and hence higher soot or $c 0$ yields) when the radiant losses cause quenching of gaseous reactions as the flame temperature becomes less than about $1500^{\circ} \mathrm{K}$. We promise to return to this subject in a later publication.

In conclusion, we again emphasize that species concentration (of $\mathrm{co}$, for example) depend for the same fuel on a) thermochemical conditions (e.8. vitiation, heating), b) radiant losses, and c) the level of turbulent flow fluctuations (e.g. free fire plume, wall plume, ceiling layer). These effects may explain the wide variation of $\mathrm{CO}$ concentration for the same fuel observed in applications.

\section{REFERENCES}

1. Orloff, L., de Ris, J., and Delichatsios, M.A., "Chemical Modeling of Gaseous Species in Turbulent Fires," Factory Mutual Research Report, August 1985, published in two parts a) 21 st Symposium on Combustion, 1986, b) Combustion and Flame, Vol 69, No. 3, Sept. 1987, pp 273-290.

2. Bilger, R.W., Combustion and Flame, 30, 277 (1977).

3. Mitchell, R.E., Sarofim, A.F., and Clomburg, L.A., Combustion and Flame, 37, 227 (1980).

4. Pope, S.B., "Paf Methods for Turbulent Reactive Flows," Progress in Energy and Combustion Science, Vol. II, 2, 119 (1985).

5. Gengembre, E., "Contributions a l'etude des Flammes de Difrusion Turbulentes a bas Nombre de Froude," Ph.D. Thesis, University of Poitiers (1983).

6. Delichatsios, M.A. and Orloff, L., "Entrainment Measurements in Turbulent Jet Flames and Implications for Modeling," FMRC Report 1984, 20th International Symposium on Combustion, 1985.

7. Delichatsios, M.A., "Air Entrainment in Turbulent Fires," Combustion and Flame, Vol. 70, No. 1, pp 33-46 (1987), oct. 1989.

8. Steward, F.R., "Predictions of the Height of Turbulent Diffusion Flames," Combustion Science and Technology 2, 203, 1970.

9. Tamanini, F., "An Integral Model of Turbuient Fire Plumes," Eighteenth (International) Symposium on Combustion, 1981. 
10. "Evaluation of Data on Simple Turbulent Reacting Flows," Edited W.C. Strahle and S.G. Lecoudis, AFOSR TR-850880, September 1985.

11. Delichatsios, M.A. and Orloff, L.: "The Effects of Turbulence Flame Radiation from Turbulent Diffusion Jet Flames," for present tion at the Eastern Section Meeting of the Combustion Institut National Bureau of Standards, Nov. 1987.

12. Drake, M.C., "Kinetics of Nitric Oxide Formation in Laminar a Turbulent Methane Combustion," Gas Research Institute Report, GF $85 / 0271$, December 1985.

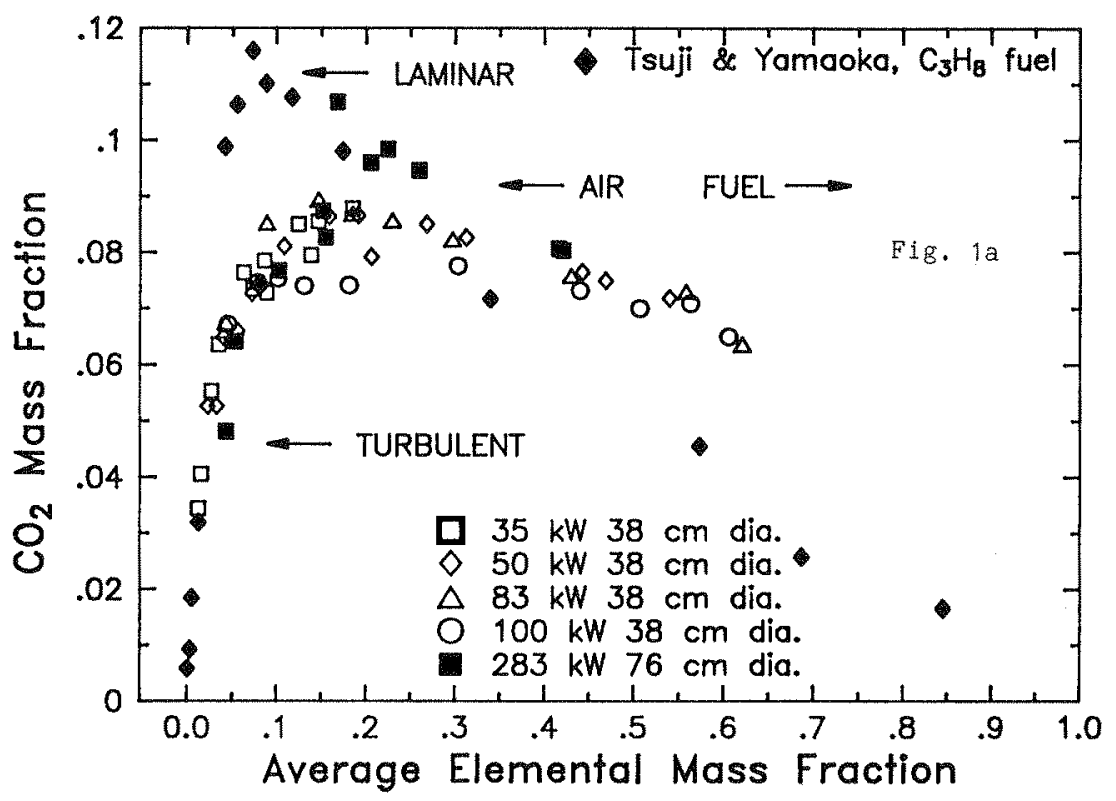



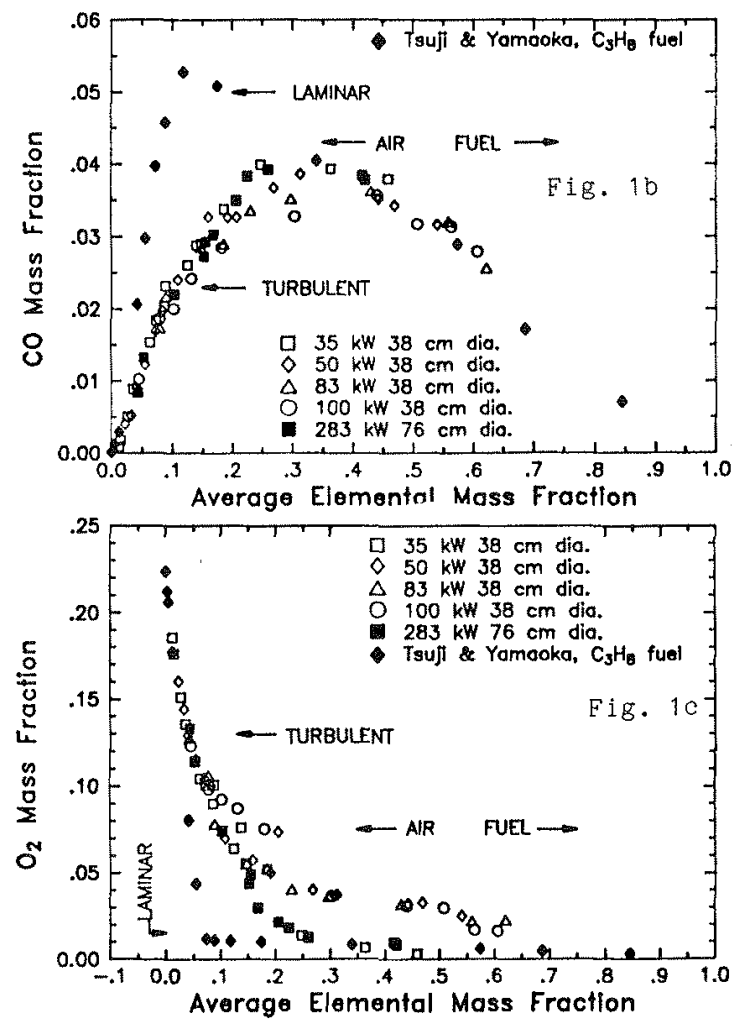

Figure $1 \mathrm{a}, \mathrm{b}, \mathrm{c}-\mathrm{CO}_{2}, \mathrm{CO}$ and $\mathrm{O}_{2}$ mean mass fractions in turbulent propane fires correlated in terms of the local mixture fraction at various axial and radial positions inside the flame. The corresponding laminar concentrations are also shown for the Tsuji and Yamaoka flame.

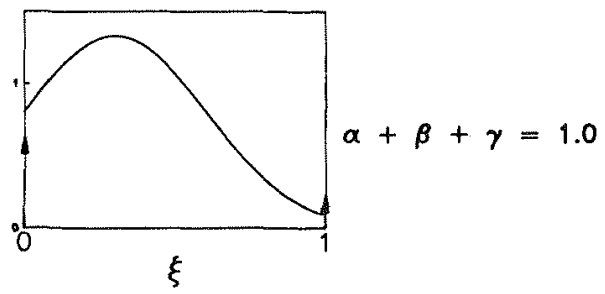

$$
\varepsilon=\frac{\overline{\xi^{2}}}{\bar{\xi}(1-\bar{\xi})} \leq 1
$$

$$
P(\xi)=\alpha \delta(\xi)+\beta P_{t}(\xi)+\gamma \delta(1-\xi)
$$

Figure 2 - A sketch of a general two-parameter probability distribution function for the mixture fraction in turbulent fires. 

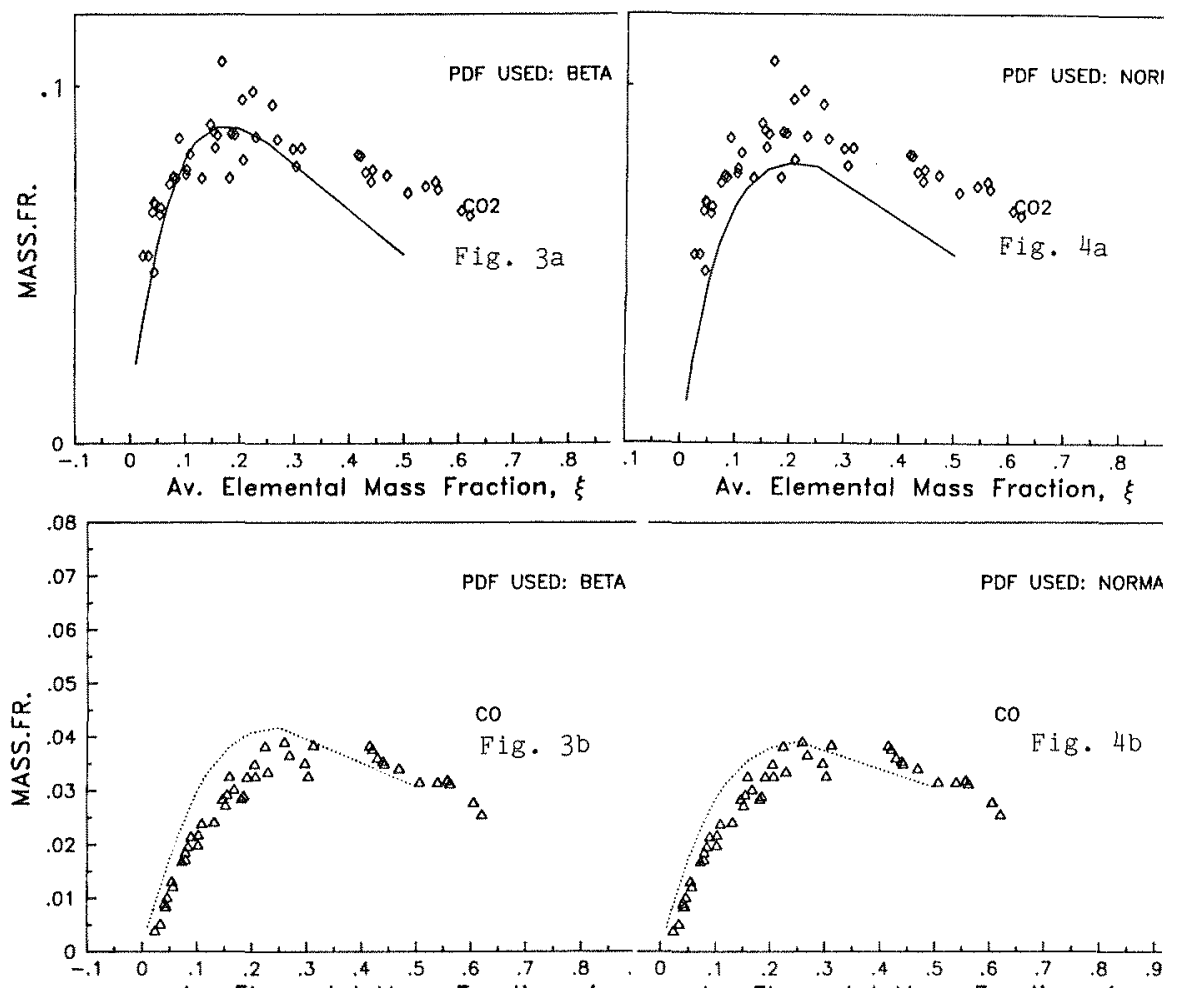

Av. Elemental Mass Fraction, $\xi$

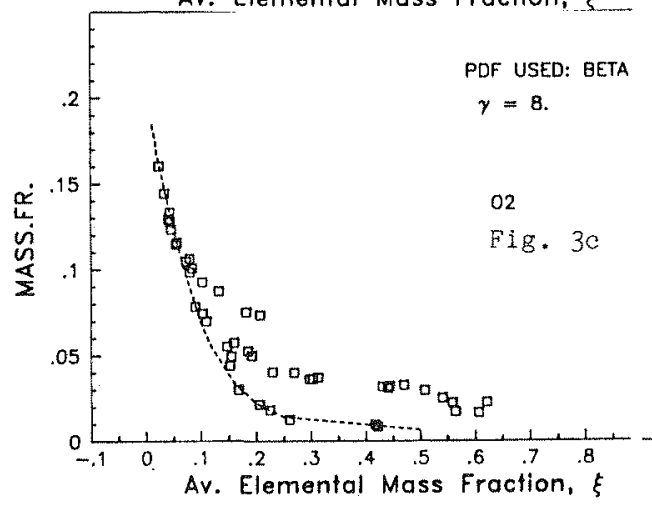

$\mathrm{Fig} \cdot 4 \mathrm{~b}$
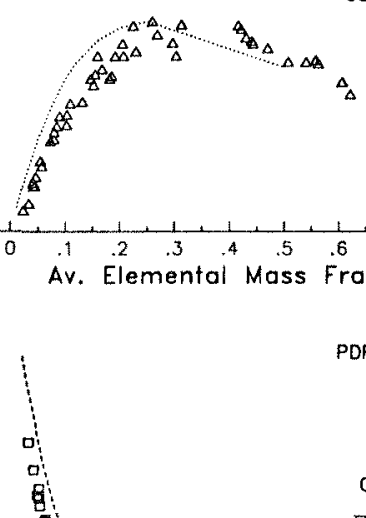

PDF USED: NORM

Figure 3 a,b,c - Calculated mass fractions of $\mathrm{CO}_{2}, \mathrm{CO}$ and $\mathrm{O}_{2}$ in turbulent fires by using a beta probability distribution function for the mixture fraction having a mixedness parameter $\varepsilon=.11$.

The experimental data of Figure 1 are also shown for comparison. 